\title{
On Type of Periodicity and Ergodicity to a Class of Fractional Order Differential Equations
}

\author{
Ravi P. Agarwal, ${ }^{1,2}$ Bruno de Andrade, ${ }^{3}$ and Claudio Cuevas ${ }^{3}$ \\ ${ }^{1}$ Department of Mathematical Sciences, Florida Institute of Technology, Melbourne, FL 32901, USA \\ ${ }^{2}$ Mathematics and Statistics Department, King Fahd University of Petroleum and Minerals, \\ Dhahran 31261, Saudi Arabia \\ ${ }^{3}$ Departamento de Matemática, Universidade Federal de Pernambuco, Recife-PE, CEP. 50540-740, Brazil \\ Correspondence should be addressed to Ravi P. Agarwal, agarwal@fit.edu
}

Received 22 October 2009; Accepted 27 January 2010

Academic Editor: Mouffak Benchohra

Copyright (C) 2010 Ravi P. Agarwal et al. This is an open access article distributed under the Creative Commons Attribution License, which permits unrestricted use, distribution, and reproduction in any medium, provided the original work is properly cited.

We study several types of periodicity to a class of fractional order differential equations.

\section{Introduction}

Fractional order differential equations is a very important subject matter. These orders can be complex in viewpoint of pure mathematics. During the last few decades fractional order differential equations have emerged vigorously (cf., [1-8]). We observe that there is much interest in developing the qualitative theory of such equations. Indeed, this has been strongly motivated by their natural and widespread applicability in several fields of sciences and technology. Many real phenomena in those fields can be described very successfully by models using mathematical tools of fractional calculus, such as dielectric polarization, electrode-electrolyte polarization, electromagnetic wave, modeling of earthquake, fluid dynamics, traffic model with fractional derivative, measurement of viscoelastic material properties, modeling of viscoplasticity, Control Theory, and economy (cf., [3, 4, 9-15]). Very recently, some basic theory for initial value problem of fractional differential equations involving the Riemann-Liouville differential operators was discussed by Benchohra et al. [16], Agarwal et al. [17-19], Lakshmikantham [20], and Lakshmikantham and Vatsala $[21,22]$. Mophou and N'Guérékata [23] have studied existence of mild solution for fractional semilinear differential equations with nonlocal conditions (more details can be found in [2429]). El-Sayed and Ibrahim [30] and Benchohra et al. [31] initiated the study of fractional multivalued differential inclusions. In this direction, we refer to the article by Henderson and Ouahab [32] concerning the existence of solutions to fractional functional differential 
inclusions with finite delay, and existence of solutions for these types of equations in the infinite delay framework (see $[16,31]$ ). In the case that fractional order is $\alpha \in(1,2]$, existence results for fractional boundary value problems of differential inclusions were studied by Ouahab [33].

We study in this work some sufficient conditions for the existence and uniqueness of pseudo-almost periodic mild solutions to the following semilinear fractional differential equation:

$$
D_{t}^{\alpha} u(t)=A u(t)+D_{t}^{\alpha-1} f(t, u(t)), \quad t \in \mathbb{R},
$$

where $1<\alpha<2, A: D(A) \subset X \rightarrow X$ is a linear densely defined operator of sectorial type on a complex Banach space $X$, and $f: \mathbb{R} \times X \rightarrow X$ is a pseudo-almost periodic function (see Definition 2.10) satisfying suitable conditions in $x$. The fractional derivative is understood in the Riemann-Liouville sense. Type (1.1) equations are attracting increasing interest. For example, anomalous diffusion in fractals by Eidelman and Kochubei [10] or in macroeconomics by Ahn and McVinisch [1] has been recently studied in the setting of fractional differential equations like (1.1). The study of almost automorphic mild solutions of (1.1) was studied by Cuevas and Lizama in [34] (see also [35]).

As for almost periodic functions, pseudo-almost periodic functions have many applications in several problems, for example, in theory of functional differential equations, integral equations, and partial differential equations. The concept of pseudo-almost periodic was introduced by Zhang [36-39] in the early nineties. Since then, such notion became of great interest to several mathematicians (see [40-49]). To the knowledge of the authors, no results yet exist for pseudo-almost periodic mild solution of (1.1).

We also discuss sufficient conditions for the existence and uniqueness of an asymptotically almost periodic mild solution of the fractional Cauchy problem

$$
\begin{gathered}
v^{\prime}(t)=\int_{0}^{t} \frac{(t-s)^{\alpha-2}}{\Gamma(\alpha-1)} A v(s) d s+f(t, v(t)), \quad t \geq 0, \\
v(0)=u_{0} \in X .
\end{gathered}
$$

In a work by Cuevas and de Souza [50] the authors proved existence and uniqueness of an S-asymptotically $\omega$-periodic solution of problem (1.2)-(1.3) (see also [51]). On the other hand, we give results on existence and uniqueness of an asymptotically almost automorphic mild solution to a class of fractional integrodifferential neutral equations.

We now turn to a summary of this work. The second section provides the definitions and preliminaries results to be used in theorems stated and proved in this article. In particular, we review some of the standard properties of the solution operator generated by a sectorial operator (see Proposition 2.2). We also recall the notion of almost periodicity, asymptotically almost periodicity, asymptotically almost automorphy, and pseudo-almost periodicity. In the third section, we obtain very general results on the existence of pseudo-almost periodic mild solution to equation (1.1). The fourth section is concerned with the existence of an asymptotically almost periodic mild solution to problem (1.2)-(1.3). While in the fifth section we use the machinery developed in the previous sections to obtain new results on existence and uniqueness of an asymptotically almost automorphic solution to a class of fractional integrodifferential neutral equation. To build intuition and throw some light on the power of our results and methods, we give, in the sixth section, a few applications. 


\section{Preliminaries and Basic Results}

Let $(Z,\|\cdot\|)$ and $(W,\|\cdot\|)$ be two Banach spaces. The notation $C(\mathbb{R}, Z)$ and $B C(\mathbb{R}, Z)$ stand for the collection of all continuous functions from $\mathbb{R}$ into $Z$ and the Banach space of all bounded continuous functions from $\mathbb{R}$ into $Z$ endowed with the uniform convergence topology, respectively. Similarly, $C(\mathbb{R} \times Z, W)$ and $B C(\mathbb{R} \times Z, W)$ stand, respectively, for the class of all jointly continuous functions from $\mathbb{R} \times Z$ into $W$ and the collection of all jointly bounded continuous functions from $\mathbb{R} \times Z$ into $W$. The notation $\mathcal{L}(Z, W)$ stands for the space of bounded linear operators from $Z$ into $W$ endowed with the uniform operator topology, and we abbreviate it to $\mathcal{L}(Z)$ whenever $Z=W$. We set $B_{r}(Z)$ for the closed ball with center at 0 radius $r$ in the space $Z$. A closed and linear operator $A$ is said to be sectorial of type $\mu$ if there exist $0<\theta<\pi / 2, M>0$ and $\mu \in \mathbb{R}$ such that its resolvent exists outside the sector $\mu+S_{\theta}:=\{\mu+\lambda: \lambda \in \mathbb{C},|\arg (-\lambda)|<\theta\}$ and $\left\|(\lambda-A)^{-1}\right\| \leq M /|\lambda-\mu|, \lambda \notin \mu+S_{\theta}$. Sectorial operators are well studied in the literature. For a recent reference including several examples and properties we refer the reader to [52]. In order to give an operator theoretical approach, we recall the following definition (cf., $[50,51])$.

Definition 2.1. Let $A$ be a closed and linear operator with domain $D(A)$ defined on a Banach space $X$. Recall $A$ the generator of a solution operator if there exist $\mu \in \mathbb{R}$ and a strongly continuous function $S_{\alpha}: \mathbb{R}_{+} \rightarrow \mathcal{L}(X)$ such that $\left\{\lambda^{\alpha}: \operatorname{Re} \lambda>\mu\right\} \subset \rho(A)$ and $\lambda^{\alpha-1}\left(\lambda^{\alpha}-A\right)^{-1} x=$ $\int_{0}^{\infty} e^{-\lambda t} S_{\alpha}(t) x d t, \operatorname{Re} \lambda>\mu, x \in X$. In this case, $S_{\alpha}(t)$ is called the solution operator generated by $A$.

We note that if $A$ is sectorial of type $\mu$ with $0 \leq \theta<\pi(1-\alpha / 2)$, then $A$ is the generator of a solution operator given by $S_{\alpha}(t):=1 / 2 \pi i \int_{\gamma} e^{\lambda t} \lambda^{\alpha-1}\left(\lambda^{\alpha}-A\right)^{-1} d \lambda$, where $\gamma$ is a suitable path lying outside the sector $\mu+S_{\theta}$ (cf., Cuesta's paper [53]). Very recently, Cuesta [53, Theorem 1] has proved that if $A$ is a sectorial operator of type $\mu<0$ for some $M>0$ and $0 \leq \theta<\pi(1-\alpha / 2)$, then there exists $C>0$ such that

$$
\left\|S_{\alpha}(t)\right\|_{\mathcal{L}(X)} \leq \frac{C M}{1+|\mu| t^{\alpha}}, \quad t \geq 0
$$

Note that $S_{\alpha}(t)$ is, in fact, integrable. The concept of a solution operator, as defined above, is closely related to the concept of a resolvent family (see Prüss [54, Chapter 1]). For the scalar case, where there is a large bibliography, we refer the reader to the monography by Gripenberg et al. [55] and references therein. Because of the uniqueness of the Laplace transform, in the border case $\alpha=1$, the family $S_{\alpha}(t)$ corresponds to a $C_{0}$-semigroup, whereas in the case $\alpha=2$ a solution operator corresponds to the concept of a cosine family; see Arendt et al. [56] and Fattorini [57]. We note that solution operators, as well as resolvent families, are a particular case of $(a, k)$-regularized families introduced by Lizama [58]. According to [58] a solution operator $S_{\alpha}(t)$ corresponds to a $\left(1, t^{\alpha-1} / \Gamma(\alpha)\right)$-regularized family. The following result is a direct consequence of [58, Proposition 3.1 and Lemma 2.2].

Proposition 2.2. Let $S_{\alpha}(t)$ be a solution operator on $X$ with generator $A$. Then, one has the following.

(a) $S_{\alpha}(t) D(A) \subset D(A)$ and $A S_{\alpha}(t) x=S_{\alpha}(t) A x$ for all $x \in D(A), t \geq 0$.

(b) Let $x \in D(A)$ and $t \geq 0$. Then $S_{\alpha}(t) x=x+\int_{0}^{t}\left((t-s)^{\alpha-1} / \Gamma(\alpha)\right) A S_{\alpha}(s) d s$.

(c) Let $x \in X$ and $t \geq 0$. Then $\int_{0}^{t}\left((t-s)^{\alpha-1} / \Gamma(\alpha)\right) S_{\alpha}(s) x d s \in D(A)$,

$$
S_{\alpha}(t) x=x+A \int_{0}^{t} \frac{(t-s)^{\alpha-1}}{\Gamma(\alpha)} S_{\alpha}(s) x d s
$$


A characterization of generators of solution operators, analogous to the Hille-Yosida Theorem for $C_{0}$-semigroup, can be directly deduced from [58, Theorem 3.4]. Results on perturbation, approximation, representation as well as ergodic type theorems can be deduced from the more general context of $(a, k)$-regularized resolvents (see [58-61]).

Let us recall the notions of almost periodicity, asymptotically almost periodicity, asymptotically almost automorphy, and pseudo-almost periodicity which shall come into play later on.

Definition 2.3 (see [62]). Let $(X,\|\cdot\|)$ be a Banach space. Then $f: \mathbb{R} \rightarrow X$ is called almost periodic if $f$ is continuous, and for each $\epsilon>0$ there exists $l(\epsilon)>0$ such that for every interval of length $l(\epsilon)$ it contains a number $\tau$ with the property that $\|f(t+\tau)-f(t)\| \leq \epsilon$ for each $t \in \mathbb{R}$. The number $\tau$ above is called an $\epsilon$-translation number for $f$, and the collection of such functions will be denoted by $A P(X)$.

Remark 2.4 (see [63]). Note that each almost periodic function is bounded and uniformly continuous. It is well known that the range $\mathcal{R}_{f}=\{f(t): t \in \mathbb{R}\}$ of an almost periodic function $f$ is relatively compact. $A P(X)$ endowed with the norm of uniform convergence on $\mathbb{R}$ is a Banach space.

Definition 2.5. Let $X$ and $Y$ be two Banach spaces. Then $f: \mathbb{R} \times Y \rightarrow X$ is called almost periodic in $t$ uniformly for $x \in Y$ if $f$ is continuous, and for each $\epsilon>0$ and any compact $K \subset Y$ there exists $l(\epsilon)>0$ such that every interval $I$ of length $l(\epsilon)$ it contains a number $\tau$ with the property that $\|f(t+\tau, x)-f(t, x)\| \leq \epsilon$ for all $t \in \mathbb{R}, x \in K$. The collection of such functions will be denoted by $A P(\mathbb{R} \times Y, X)$.

It is well known that the study of composition of two functions with special properties is important and basic for deep investigations. We begin with the following standard result in the theory of almost periodic function (see $[39,63])$.

Lemma 2.6. Let $f \in A P(\mathbb{R} \times Y, X)$ and $h \in A P(Y)$. Then the function $f(\cdot, h(\cdot)) \in A P(X)$.

Definition 2.7. A continuous function $f:[0, \infty) \rightarrow X$ (resp., $[0, \infty) \times Y \rightarrow X$ ) is called asymptotically almost periodic (resp., asymptotically almost periodic in $t$ uniformly in $y \in Y$ ) if it admits a decomposition $f=g+\phi$, where $g \in A P(X)$ (resp., $g \in A P(\mathbb{R} \times Y, X)$ ) and $\phi \in C_{0}([0, \infty), X)$ (resp., $\left.\phi \in C_{0}([0, \infty) \times Y, X)\right)$. Here $C_{0}([0, \infty), X)$ denotes the subspace of $B C([0, \infty), X)$ such that $\lim _{t \rightarrow \infty}\|x(t)\|=0$ and $C_{0}([0, \infty) \times Y, X)$ denotes the space of all continuous functions $h:[0, \infty) \times Y \rightarrow X$ such that $\lim _{t \rightarrow \infty} h(t, x)=0$ uniformly for $x$ in any compact subset of $Y$. Denote by $A A P(X)$ (resp., $A A P(Y, X)$ ) the set of all such functions. $A A P(X)$ is a Banach space with the sup norm.

Definition 2.8. A continuous function $f:[0, \infty) \times Y \rightarrow X$ is called uniformly continuous on bounded sets uniformly for $t \geq 0$ if for every $\epsilon>0$ and every bounded subset $K$ of $Y$ there exists $\delta_{\epsilon, K}>0$ such that $\|f(t, x)-f(t, y)\| \leq \epsilon$ for all $t \geq 0$ and all $x, y \in K$ so that $\|x-y\| \leq \delta_{\epsilon, K}$.

Lemma 2.9. Let $f \in A A P(Y, X)$ and let $f(t, y)$ be uniformly continuous on bounded sets uniformly for $t \geq 0$. If $u \in A A P(Y)$, then $f(\cdot, u(\cdot)) \in A A P(X)$.

Let $P A P_{0}(X)$ denote the space of all bounded continuous functions $\Phi: \mathbb{R} \rightarrow X$ such that

$$
\lim _{r \rightarrow \infty} \frac{1}{2 r} \int_{-r}^{r}\|\Phi(t)\| d t=0
$$


and $P A P_{0}(\mathbb{R} \times Y, X)$ denotes the space of all continuous functions such that $\Phi(\cdot, x)$ is bounded for all $x \in Y$ and

$$
\lim _{r \rightarrow \infty} \frac{1}{2 r} \int_{-r}^{r}\|\Phi(t, x)\| d t=0
$$

uniformly in $x \in Y$.

Definition 2.10 (see $[36,64]$ ). A function $f \in B C(\mathbb{R}, X)$ (resp, $f \in B C(\mathbb{R} \times Y, X)$ ) is called pseudo-almost periodic (resp., pseudo-almost periodic in $t \in \mathbb{R}$ uniformly in $x \in Y$ ) if $f=$ $g+\Phi$ where $g \in A P(X)(A P(\mathbb{R} \times Y, X))$ and $\Phi \in P A P_{0}(X)\left(P A P_{0}(\mathbb{R} \times Y, X)\right)$.

The functions $g$ and $\Phi$ are called the almost periodic component and, respectively, the ergodic perturbation of the function $f$. The set of all such functions will be denoted by $P A P(X)$ (resp., $P A P(\mathbb{R} \times Y, X)$ ). Obviously $P A P(X)$ is a subspace of $B C(\mathbb{R}, X)$. Furthermore, we have that $P A P(X)$ is a closed subspace of $B C(\mathbb{R}, X)$; hence, it is a Banach space with the supremum norm (see [65]).

Lemma 2.11 (see [65]). Let $f \in P A P(\mathbb{R} \times Y, X)$ satisfy the following conditions.

(i) $\{f(t, x): t \in \mathbb{R}$ and $x \in K\}$ is bounded for every bounded subset $K \subset Y$.

(ii) $f(t, \cdot)$ is uniformly continuous in each bounded subset of $Y$ uniformly in $t \in \mathbb{R}$. More explicitly, given $\epsilon>0$ and $K \subset Y$ bounded, there exists $\delta>0$ such that $x, y \in K$ and $\|x-y\| \leq \delta$ imply that $\|f(t, x)-f(t, y)\| \leq \epsilon$ for all $t \in \mathbb{R}$.

If $h \in P A P(Y)$, then $f(\cdot, h(\cdot)) \in P A P(X)$.

Lemma 2.12. Assume that $A$ is sectorial of type $\mu<0$. If $g: \mathbb{R} \rightarrow X$ is an almost periodic function and $\Gamma g$ is given by

$$
(\Gamma g)(t)=\int_{-\infty}^{t} S_{\alpha}(t-s) g(s) d s, \quad t \in \mathbb{R}
$$

then $\Gamma g \in A P(X)$.

Proof. For $\epsilon>0$, we take $l(\epsilon)$ involved in Definition 2.3, then for every interval of length $l(\epsilon)$ contains a number $\tau$ such that $\|g(t+\tau)-g(t)\| \leq \epsilon$ for each $t \in \mathbb{R}$. The estimate

$$
\begin{aligned}
\|\Gamma g(t+\tau)-\Gamma g(t)\| & \leq \int_{0}^{\infty}\left\|S_{\alpha}(s)\right\|\|g(t-s-\tau)-g(t-s)\| d s \\
& \leq\left(C M \int_{0}^{\infty} \frac{1}{1+|\mu| s^{\alpha}} d s\right) \epsilon=\frac{C M|\mu|^{-1 / \alpha} \pi \epsilon}{\alpha \sin (\pi / \alpha)}
\end{aligned}
$$

is responsible for the fact that $\Gamma g \in A P(X)$. 
Lemma 2.13. Assume that $A$ is sectorial of type $\mu<0$. If $f:[0, \infty) \rightarrow X$ is an asymptotically almost periodic function and $\Gamma^{*} f$ is given by

$$
\left(\Gamma^{*} f\right)(t)=\int_{0}^{t} S_{\alpha}(t-s) f(s) d s, \quad t \geq 0,
$$

then $\Gamma^{*} f \in A A P(X)$.

Proof. If $f=g+\Phi$, where $g \in A P(X)$ and $\Phi \in C_{0}([0, \infty), X)$, then we have that $\Gamma^{*} f(t)=$ $G^{*}(t)+H^{*}(t)$, where

$$
\begin{gathered}
G^{*}(t):=\int_{-\infty}^{t} S_{\alpha}(t-s) g(s) d s \quad t \in \mathbb{R}, \\
H^{*}(t):=\int_{0}^{t} S_{\alpha}(t-s) \Phi(s) d s-\int_{-\infty}^{0} S_{\alpha}(t-s) g(s) d s .
\end{gathered}
$$

By the previous lemma $G^{*} \in A P(X)$. Next, let us show that $H^{*} \in C_{0}([0, \infty), X)$. Since $\Phi \in$ $C_{0}([0, \infty), X)$, for each $\epsilon>0$ there exists a constant $T>0$ such that $\|\Phi(s)\| \leq \epsilon$ for all $s \geq T$. Then for all $t \geq 2 T$, we deduce

$$
\begin{aligned}
\left\|H^{*}(t)\right\| \leq & C M\|\Phi\|_{\infty} \int_{0}^{t / 2} \frac{1}{1+|\mu|(t-s)^{\alpha}} d s+\epsilon C M \int_{t / 2}^{t} \frac{1}{1+|\mu|(t-s)^{\alpha}} d s \\
& +C M\|g\|_{\infty} \int_{-\infty}^{0} \frac{1}{1+|\mu|(t-s)^{\alpha}} d s \\
\leq & C M\left(\|\Phi\|_{\infty}+\|g\|_{\infty}\right) \int_{t}^{\infty} \frac{1}{1+|\mu| s^{\alpha}} d s+\frac{\epsilon C M|\mu|^{-1 / \alpha} \pi}{\alpha \sin (\pi / \alpha)} .
\end{aligned}
$$

Therefore, $\lim _{t \rightarrow \infty} H^{*}(t)=0$, that is, $H^{*} \in C_{0}([0, \infty), X)$. This completes the proof.

Lemma 2.14. Assume that $A$ is sectorial of type $\mu<0$. If $f: \mathbb{R} \rightarrow X$ is pseudo-almost periodic function and $\Gamma f$ is the function defined in (2.5). Then $\Gamma f \in P A P(X)$.

Proof. It is clear that $\Gamma f \in B C(\mathbb{R}, X)$. In fact, we get $\|\Gamma f\|_{\infty} \leq\left(C M|\mu|^{-1 / \alpha} \pi\|f\|_{\infty}\right) /(\alpha \sin (\pi / \alpha))$, where $C$ and $M$ are given by (2.1). If $f=g+\Phi$, where $g \in A P(X)$ and $\Phi \in P A P_{0}(X)$, then from Lemma 2.12, $\Gamma g \in A P(X)$. To complete the proof, we show that $\Gamma \Phi \in P A P_{0}(X)$. For $r>0$ we see that

$$
\frac{1}{2 r} \int_{-r}^{r}\left\|\int_{0}^{\infty} S_{\alpha}(s) \Phi(t-s) d s\right\| d t \leq \frac{1}{2 r} \int_{-r}^{r} \int_{0}^{\infty}\left\|S_{\alpha}(s)\right\|\|\Phi(t-s)\| d s d t \leq C M \int_{0}^{\infty} \frac{\Phi_{r}(s)}{1+|\mu|^{\alpha}} d s,
$$

where $\Phi_{r}(s)=(1 / 2 r) \int_{-r}^{r}\|\Phi(t-s)\| d t, s \geq 0$. 
It is not hard to check that $\Phi_{r}(t) \rightarrow 0$ as $r \rightarrow \infty$. Next, since $\Phi_{r}(s)$ is bounded and $1 /\left(1+|\mu| s^{\alpha}\right)$ is integrable in $[0, \infty)$, using the Lebesgue dominated convergence theorem, it follows that $\lim _{r \rightarrow \infty} \int_{0}^{\infty}\left(\Phi_{r}(s) /\left(1+|\mu| s^{\alpha}\right)\right) d s=0$. The proof is now completed.

Let $h: \mathbb{R} \rightarrow[1, \infty)$ be a continuous function such that $h(t) \rightarrow \infty$ as $|t| \rightarrow \infty$. We consider the space $C_{h}(X)=\left\{u \in C(\mathbb{R}, X): \lim _{|t| \rightarrow \infty}(u(t) / h(t))=0\right\}$ endowed with the norm $\|u\|_{h}=\sup _{t \in \mathbb{R}}\|u(t)\| / h(t)$.

Lemma 2.15 (see [66]). A subset $K \subseteq C_{h}(X)$ is a relatively compact set if it verifies the following conditions.

$(c-1)$ The set $K(t)=\{u(t): u \in K\}$ is relatively compact in $X$ for each $t \in \mathbb{R}$.

(c-2) The set $K$ is equicontinuous.

(c-3) For each $\epsilon>0$ there exists $L>0$ such that $\|u(t)\| \leq \epsilon h(t)$ for all $u \in K$ and all $|t|>L$.

Let $h^{*}:[0, \infty) \rightarrow[1, \infty)$ be a continuous function such that $h^{*}(t) \rightarrow \infty$ as $t \rightarrow \infty$. Consider the space $C_{h^{*}}(X)=\left\{u \in C([0, \infty), X): \lim _{t \rightarrow \infty}\left(u(t) / h^{*}(t)\right)=0\right\}$ endowed with the norm $\|u\|_{h^{*}}=\sup _{t \geq 0}\left(\|u(t)\| / h^{*}(t)\right)$.

Lemma 2.16 (see [67]). A subset $K \subseteq C_{h^{*}}(X)$ is a relatively compact set if it verifies the following conditions.

(c-1) The set $K_{b}=\left\{\left.u\right|_{[0, b]}: u \in K\right\}$ is relatively compact in $C([0, b] ; X)$ for all $b \geq 0$.

$(c-2) \lim _{t \rightarrow \infty}(\|u(t)\|) /\left(h^{*}(t)\right)=0$ uniformly for all $u \in K$.

Definition 2.17. A continuous function $f: \mathbb{R} \rightarrow X$ is called almost automorphic if for every sequence of real numbers $\left(s_{m}\right)_{m \in \mathbb{N}}$ there exists a subsequence $\left(s_{n}\right)_{n \in \mathbb{N}}$ such that $g(t)=$ $\lim _{n \rightarrow \infty} f\left(t+s_{n}\right)$ is well defined for each $t \in \mathbb{R}$, and $f(t)=\lim _{n \rightarrow \infty} g\left(t-s_{n}\right)$ for each $t \in \mathbb{R}$. Denote by $A A(\mathbb{R}, X)$ the set of all such functions; it constitutes a Banach space when it is endowed with the sup norm.

Almost automorphic functions were introduced by Bochner [68] as a natural generalization of the concept of almost periodic function. A complete description of the properties and further applications to evolution equations can be found in the monographs [69] and [70] by N'Guérékata.

Definition 2.18. Let $X$ and $Y$ be two Banach spaces. A continuous function $f: \mathbb{R} \times Y \rightarrow X$ is called almost automorphic in $t$ uniformly for $x$ in compact subsets of $Y$ if for every compact subset $K$ of $Y$ and every real sequence $\left(s_{m}\right)_{m \in \mathbb{N}}$ there exists a subsequence $\left(s_{n}\right)_{n \in \mathbb{N}}$ such that $\tilde{g}(t, x)=\lim _{n \rightarrow \infty} f\left(t+s_{n}, x\right)$ is well defined for each $t \in \mathbb{R}, x \in K$, and $\lim _{n \rightarrow \infty} \widetilde{g}\left(t-s_{n}, x\right)=$ $f(t, x)$ for each $t \in \mathbb{R}, x \in K$. Denote by $A A(\mathbb{R} \times Y, X)$ the set of all such functions.

Lemma 2.19 (see [34]). Assume that $A$ is sectorial of type $\mu<0$. If $g: \mathbb{R} \rightarrow X$ is an almost automorphic function and $\Gamma g$ is given by (2.5), then $\Gamma g \in A A(\mathbb{R}, X)$.

In 1980s, N'Guérékata [71] defined asymptotically almost automorphic functions as perturbation of almost automorphic functions by functions vanishing at infinite. Since then, those functions have generated lots of developments and applications; we refer the reader to $[69,72-74]$ and the references therein. 
Definition 2.20 (see [75]). A continuous function $f:[0, \infty) \rightarrow X($ resp., $[0, \infty) \times Y \rightarrow$ $X$ ) is called asymptotically almost automorphic (asymptotically almost automorphic in $t$ uniformly for $x$ in compact subsets of $Y$ ) if it admits a decomposition $f=g+\Phi, t \geq 0$, where $g \in A A(\mathbb{R}, X)$ (resp., $A A(\mathbb{R} \times Y, X))$ and $\Phi \in C_{0}([0, \infty), X)\left(\right.$ resp., $\left.C_{0}([0, \infty) \times Y, X)\right)$. Denote by $A A A([0, \infty), X)$ (resp., $A A A([0, \infty) \times Y, X))$ the set of all such functions. $A A A([0, \infty), X)$ is a Banach space with the sup norm (see [75, Lemma 1.8]). We note that the range of an asymptotically almost automorphic function is relatively compact [75].

Lemma 2.21. Assume that $A$ is sectorial of type $\mu<0$. If $f:[0, \infty) \rightarrow X$ is an asymptotically almost automorphic function and $\Gamma^{*} f$ is given by $(2.7)$, then $\Gamma^{*} f \in A A A([0, \infty), X)$.

Proof. $f=g+\Phi$, where $g \in A A(\mathbb{R}, X)$ and $\Phi \in C_{0}([0, \infty), X)$. We have that $\Gamma^{*} f=G^{*}+H^{*}$, where $G^{*}$ and $H^{*}$ are the functions given by (2.8) and (2.9), respectively. By previous lemma $G^{*} \in A A(\mathbb{R}, X)$ and by the proof of Lemma $2.13 H^{*} \in C_{0}([0, \infty), X)$. This ends the proof.

Lemma 2.22 (see [75]). Let $f \in A A A([0, \infty) \times Y, X)$ and let $f(t, y)$ be uniformly continuous on bounded sets uniformly for $t \geq 0$. If $u \in A A A([0, \infty), Y)$, then $f(\cdot, u(\cdot)) \in A A A([0, \infty), X)$.

\section{Pseudo-Almost Periodic Mild Solutions}

We recall the following definition that will be essential for us.

Definition 3.1 (see [34]). Suppose that $A$ generates an integrable solution operator $S_{\alpha}(t)$. A continuous function $u: \mathbb{R} \rightarrow X$ satisfying the integral equation

$$
u(t)=\int_{-\infty}^{t} S_{\alpha}(t-s) f(s, u(s)) d s, \quad \forall t \in \mathbb{R}
$$

is called a mild solution to the equation (1.1).

The following are the main results of this section.

Theorem 3.2. Assume that $A$ is sectorial of type $\mu<0$. Let $f: \mathbb{R} \times X \rightarrow X$ be a function pseudoalmost periodic in $t \in \mathbb{R}$, uniformly in $x \in X$, and assume that there exists an integrable bounded function $L_{f}: \mathbb{R} \rightarrow[0, \infty)$ satisfying

$$
\|f(t, x)-f(t, y)\| \leq L_{f}(t)\|x-y\|, \quad \forall x, y \in X, \forall t \in \mathbb{R}
$$

Then equation (1.1) has a unique pseudo-almost periodic mild solution.

Proof. We define the operator $F: P A P(X) \rightarrow P A P(X)$ by

$$
(F \Phi)(t)=\int_{-\infty}^{t} S_{\alpha}(t-s) f(s, \Phi(s)) d s, \quad t \in \mathbb{R} .
$$

Given $v \in P A P(X)$, in view of Lemma 2.11, we have that $s \rightarrow f(s, v(s))$ is a pseudo-almost periodic function, and hence bounded in $\mathbb{R}$. Since the function $t \rightarrow 1 /\left(1+|\mu| t^{\alpha}\right)$ is integrable 
on $[0, \infty)(1<\alpha<2)$, we get that $F v$ exists. Now, by Lemma 2.14, we obtain that $F v \in$ $P A P(X)$, and hence $F$ is well defined. It suffices to show that the operator $F$ has a unique fixed point in $P A P(X)$. For this, consider $u, v \in P A P(X)$. We can deduce that

$$
\left\|\left(F^{n} u\right)(t)-\left(F^{n} v\right)(t)\right\| \leq \frac{C_{\alpha}^{n}}{n !}\left(\int_{-\infty}^{t} L_{f}(\tau) d \tau\right)^{n}\|u-v\|_{\infty} \leq \frac{\left(C_{\alpha}\left\|L_{f}\right\|_{1}\right)^{n}}{n !}\|u-v\|_{\infty}
$$

where $C_{\alpha}:=\sup _{t \geq 0}\left\|S_{\alpha}(t)\right\|_{\mathcal{L}(X)}$. Since $\left(\left(C_{\alpha}\left\|L_{f}\right\|_{1}\right)^{n} / n !\right)<1$ for $n$ sufficiently large, by the contraction principle, $F$ has a unique fixed point $u \in P A P(X)$. This completes the proof.

We can establish the following existence result.

Proposition 3.3. Assume that $A$ is sectorial of type $\mu<0$. Let $f: \mathbb{R} \times X \rightarrow X$ be a function pseudo-almost periodic in $t \in \mathbb{R}$ uniformly in $x \in X$ that satisfies the Lipschitz condition (3.2) with $L_{f} \in B C$. Let $\left\|L_{f}\right\|=\sup _{t \in \mathbb{R}} \int_{t}^{t+1} L_{f}(s) d s$. If $C M|\mu|^{-1 / \alpha} \pi\left\|L_{f}\right\|<\alpha \sin (\pi / \alpha)$, where $C$ and $M$ are the constants in (2.1), then equation (1.1) has a unique pseudo-almost periodic mild solution.

Proof. Let $F$ be the map defined in the previous theorem. For $u, v \in P A P(X)$ we can estimate that

$$
\begin{aligned}
\|F u(t)-F v(t)\| & \leq C M \int_{-\infty}^{t} \frac{L_{f}(s)}{1+|\mu|(t-s)^{\alpha}}\|u(s)-v(s)\| d s \\
& \leq C M\left(\sum_{m=0}^{\infty} \int_{t-(m+1)}^{t-m} \frac{L_{f}(s)}{1+|\mu|(t-s)^{\alpha}} d s\right)\|u-v\|_{\infty} \\
& \leq C M\left(\sum_{m=0}^{\infty} \frac{1}{1+|\mu| m^{\alpha}} \int_{t-(m+1)}^{t-m} L_{f}(s) d s\right)\|u-v\|_{\infty} \\
& \leq C M\left(\sum_{m=0}^{\infty} \frac{1}{1+|\mu| m^{\alpha}}\right)\left\|L_{f}\right\|\|u-v\|_{\infty},
\end{aligned}
$$

which finishes the proof.

Corollary 3.4. Assume that $A$ is sectorial of type $\mu<0$. Let $f: \mathbb{R} \times X \rightarrow X$ be a function pseudoalmost periodic in $t \in \mathbb{R}$ uniformlies in $x \in X$ that satisfy the Lipschitz condition

$$
\|f(t, x)-f(t, y)\| \leq L\|x-y\|, \quad \forall x, y \in X, \forall t \in \mathbb{R} .
$$

If $C M|\mu|^{-1 / \alpha} \pi L<\alpha \sin (\pi / \alpha)$, where $C$ and $M$ are the constants given in (2.1), then equation (1.1) has a unique pseudo-almost periodic mild solution.

To establish our next result we consider perturbations $f$ of (1.1) that satisfy the following boundedness condition.

(H1) There exists a continuous nondecreasing function $W:[0, \infty) \rightarrow[0, \infty)$ such that $\|f(t, x)\| \leq W(\|x\|)$ for all $t \in \mathbb{R}$ and $x \in X$.

We have the following result. 
Theorem 3.5. Assume that $A$ is sectorial of type $\mu<0$. Let $f: \mathbb{R} \times X \rightarrow X$ be a function pseudo-almost periodic in $t \in \mathbb{R}$ uniformly in $x \in X$ that satisfies assumption (H1) and the following conditions.

(H2) $f(t, x)$ is uniformly continuous on bounded subset of $X$ uniformly in $t \in \mathbb{R}$.

(H3) For each $v \geq 0, \lim _{|t| \rightarrow \infty} 1 / h(t) \int_{-\infty}^{t}\left(W(v h(s)) /\left(1+|\mu|(t-s)^{\alpha}\right)\right) d s=0$, where $h$ is given by Lemma 2.15. Set

$$
\beta(v):=C M\left\|\int_{-\infty} \frac{W(v h(s))}{1+|\mu|(\cdot-s)^{\alpha}} d s\right\|_{h},
$$

where $C$ and $M$ are constants given in (2.1).

(H4) For each $\epsilon>0$ there is $\delta>0$ such that, for every $u, v \in C_{h}(X),\|u-v\|_{h} \leq \delta$ implies that

$$
\int_{-\infty}^{t} \frac{\|f(s, v(s))-f(s, u(s))\|}{1+|\mu|(t-s)^{\alpha}} d s \leq \epsilon
$$

for all $t \in \mathbb{R}$.

(H5) For all $a, b \in \mathbb{R}, a<b$, and $r>0$, the set $\{f(s, h(s) x): a \leq s \leq b, x \in X,\|x\| \leq r\}$ is relatively compact in $\mathrm{X}$.

(H6) $\liminf _{\xi \rightarrow \infty} \xi / \beta(\xi)>1$.

Then equation (1.1) has a pseudo-almost periodic mild solution.

Proof. We define the operator $F$ on $C_{h}(X)$ as in (3.3). We show that $F$ has a fixed point in $P A P(X)$.

(i) For $u \in C_{h}(X)$, we have that

$$
\lim _{t \rightarrow \infty} \frac{\|F u(t)\|}{h(t)} \leq \lim _{t \rightarrow \infty} \frac{C M}{h(t)} \int_{-\infty}^{t} \frac{W\left(\|u\|_{h} h(s)\right)}{1+|\mu|(t-s)^{\alpha}} d s
$$

It follows from condition (H3) that $F: C_{h}(X) \rightarrow C_{h}(X)$. From condition (H4) it follows that $F$ is a continuous map.

(ii) We next show that $F$ is completely continuous. The argument comes from Lemma 2.15. In fact, let $V=F\left(B_{r}\left(C_{h}(X)\right)\right)$ and $v=F(u)$ for $u \in B_{r}\left(C_{h}(X)\right)$. Initially, we will prove that $V(t)$ is a relatively compact subset of $X$ for each $t \in \mathbb{R}$. It follows from condition (H3) that the function $s \rightarrow W(r h(t-s)) /\left(1+|\mu| s^{\alpha}\right)$ is integrable on $[0, \infty)$. Hence, for $\epsilon>0$, we can choose $a \geq 0$ such that $C M \int_{a}^{\infty}\left(W(r h(t-s)) /\left(1+|\mu| s^{\alpha}\right)\right) d s \leq \epsilon$. Hence $v(t) \in \operatorname{ac}_{0}\left(\left\{S_{\alpha}(s) f(\xi, h(\xi) x): 0 \leq\right.\right.$ $s \leq a, t-a \leq \xi \leq t,\|x\| \leq r\})+B_{\epsilon}(X)$, where $c_{0}(K)$ denotes the convex hull of $K$. Using that $S_{\alpha}(\cdot)$ is strongly continuous and the property (H5), we infer that $K=\left\{S_{\alpha}(s) f(\xi, h(\xi) x): 0 \leq s \leq a, t-a \leq \xi \leq t,\|x\| \leq r\right\}$ is relatively compact set, and $V(t) \subset \overline{a c_{0}(K)}+B_{\epsilon}(X)$, which establishes our assertion. 
We next show that the set $V$ is equicontinuous. In fact, we can decompose

$$
\begin{aligned}
v(t+s)-v(t)= & \int_{0}^{s} S_{\alpha}(\xi) f(t+s-\xi, u(t+s-\xi)) d \xi \\
& +\int_{0}^{a}\left(S_{\alpha}(\xi+s)-S_{\alpha}(\xi)\right) f(t-\xi, u(t-\xi)) d \xi \\
& +\int_{a}^{\infty}\left(S_{\alpha}(\xi+s)-S_{\alpha}(\xi)\right) f(t-\xi, u(t-\xi)) d \xi .
\end{aligned}
$$

For each $\epsilon>0$, we can choose $a>0$ and $\delta_{1}>0$ such that

$$
\begin{gathered}
\left\|\int_{0}^{s} S_{\alpha}(\xi) f(t+s-\xi, u(t+s-\xi)) d \xi+\int_{a}^{\infty}\left(S_{\alpha}(\xi+s)-S_{\alpha}(\xi)\right) f(t-\xi, u(t-\xi)) d \xi\right\| \\
\quad \leq C M\left(\int_{0}^{s} \frac{W(r h(t+s-\xi))}{1+|\mu| \xi^{\alpha}} d \xi+2 \int_{a}^{\infty} \frac{W(r h(t-\xi))}{1+|\mu| \xi^{\alpha}} d \xi\right) \leq \epsilon / 2,
\end{gathered}
$$

for $s \leq \delta_{1}$. Moreover, since $\left\{f(t-\xi, u(t-\xi)): 0 \leq \xi \leq a, u \in B_{r}\left(C_{h}(X)\right)\right\}$ is relatively compact set and $S_{\alpha}(\cdot)$ is strongly continuous, we can choose $\delta_{2}>0$ such that $\|\left(S_{\alpha}(\xi+s)-S_{\alpha}(s)\right) f(t-$ $\xi, u(t-\xi)) \|<\epsilon / 2 a$ for $s \leq \delta_{2}$. Combining these estimates, we get $\|v(t+s)-v(t)\| \leq \epsilon$ for $s$ small enough and independent of $u \in B_{r}\left(C_{h}(X)\right)$.

Finally, applying condition (H3), we can show that

$$
\frac{v(t)}{h(t)} \leq \frac{C M}{h(t)} \int_{-\infty}^{t} \frac{W(r h(s))}{1+|\mu|(t-s)^{\alpha}} d s \longrightarrow 0, \quad|t| \longrightarrow \infty
$$

and this convergence is independent of $u \in B_{r}\left(C_{h}(X)\right)$. Taking into account Lemma 2.15, $V$ is a relatively compact set in $C_{h}(X)$.

(iii) If $u^{\lambda}(\cdot)$ is a solution of equation $u^{\lambda}=\lambda F\left(u^{\lambda}\right)$ for some $0<\lambda<1$, then we can check that $\left\|u^{\lambda}\right\|_{h} \leq \beta\left(\left\|u^{\lambda}\right\|_{h}\right)$ and, combining with condition (H6), we conclude that the set $\widetilde{K}:=\left\{u^{\lambda}: u^{\lambda}=\lambda F\left(u^{\lambda}\right), \lambda \in(0,1)\right\}$ is bounded.

(iv) It follows, from Lemmas 2.11 and 2.14, that $F(P A P(X)) \subset P A P(X)$ and, consequently, $F: P A P(X) \rightarrow P A P(X)$ is completely continuous. Since $\widetilde{K}$ is bounded and using Leray-Schauder alternative theorem, we infer that $F$ has a fixed point $u \in P A P(X)$. Let $\left(u_{n}\right)_{n}$ be a sequence in $P A P(X)$ that converges to $u$. We see that $\left(F u_{n}\right)_{n}$ converges to $F u=u$ uniformly in $\mathbb{R}$. This implies that $u \in P A P(X)$ and completes the proof.

It is particularly interesting to note that the next result is not covered by the results by Cuevas and Lizama [34].

Corollary 3.6. Assume that conditions (H1)-(H6) hold and that $A$ is sectorial of type $\mu<0$. If $f: \mathbb{R} \times X \rightarrow X$ is almost periodic in $t \in \mathbb{R}$ uniformly for $x \in X$, then equation (1.1) has an almost periodic mild solution.

Proof. It is a consequence of Lemmas 2.6 and 2.12. 


\section{Asymptotically Almost Periodic Mild Solutions}

We recall the following definition.

Definition 4.1 (see [50]). Suppose that $A$ generates an integrable solution operator $S_{\alpha}(t)$. A function $u:[0, \infty) \rightarrow X$ satisfying

$$
u(t)=S_{\alpha}(t) u_{0}+\int_{0}^{t} S_{\alpha}(t-s) f(s, u(s)) d s, \quad \forall t \geq 0
$$

is called a mild solution of the problem (1.2)-(1.3).

Theorem 4.2. Assume that $A$ is sectorial of type $\mu<0$. Let $f:[0, \infty) \times X \rightarrow X$ be a function asymptotically almost periodic in $t$ uniformly in $x \in X$ and assume that there exists an integrable bounded function $L_{f}:[0, \infty) \rightarrow[0, \infty)$ satisfying

$$
\|f(t, x)-f(t, y)\| \leq L_{f}(t)\|x-y\|, \quad \forall x, y \in X, \quad \forall t \geq 0 .
$$

Then the problem (1.2)-(1.3) has a unique asymptotically almost periodic mild solution.

Proof. We define the operator $\Gamma_{\alpha}$ on the space $A A P(X)$ by

$$
\left(\Gamma_{\alpha} u\right)(t)=S_{\alpha}(t) u_{0}+\int_{0}^{t} S_{\alpha}(t-s) f(s, u(s)) d s:=S_{\alpha}(t) u_{0}+v_{\alpha}(t)
$$

We show initially that $\Gamma_{\alpha} u \in A A P(X)$. In fact, we observe that the estimate (2.1) implies that $S_{\alpha}(\cdot) u_{0} \in A A P(X)$. It follows from Lemma 2.9 that the function $s \rightarrow f(s, u(s))$ is asymptotically almost periodic; then by Lemma 2.13, $v_{\alpha} \in A A P(X)$, and hence $\Gamma_{\alpha}$ is well defined. Let $u, v$ be in $A A P(X)$ and define $C_{\alpha}:=\sup _{t>0}\left\|S_{\alpha}(t)\right\|_{\mathcal{L}(X)}$. We have the following estimate:

$$
\left\|\Gamma_{\alpha}^{n} u-\Gamma_{\alpha}^{n} v\right\|_{\infty} \leq \frac{\left(C_{\alpha}\left\|L_{f}\right\|_{1}\right)^{n}}{n !}\|u-v\|_{\infty}
$$

which is responsible for the fact that $\Gamma_{\alpha}$ has a unique fixed point in $A A P(X)$.

Corollary 4.3. Assume that $A$ is sectorial of type $\mu<0$. Let $f:[0, \infty) \times X \rightarrow X$ be a function asymptotically almost periodic in $t$ uniformly in $x \in X$ that satisfies the Lipschitz condition (4.2) with $L_{f}(\cdot) \equiv L$. If $C M|\mu|^{-1 / \alpha} \pi L<\alpha \sin \pi / \alpha$, where $C$ and $M$ are the constants given in (2.1), then the problem (1.2)-(1.3) has a unique asymptotically almost periodic mild solution. corollary.

Taking $A=-\rho^{\alpha} I$ with $\rho>0$ and $X=\mathbb{C}$ in (1.2), the above result produces the following

Corollary 4.4. Let $f:[0, \infty) \times \mathbb{C} \rightarrow \mathbb{C}$ be a function asymptotically almost periodic in $t$ uniformly in $z \in \mathbb{C}$ that satisfies the Lipschitz condition (4.2) with $L_{f}(\cdot) \equiv L$. Then problem (1.2)-(1.3) has a unique asymptotically almost periodic solution whenever $L<(\alpha \sin \pi / \alpha) / \rho \pi$. 
Remark 4.5. A similar result as that of the previous corollary was obtained by Cuevas and de Souza [50] for obtaining an S-asymptotically $\omega$-periodic mild solution for problem (1.2)-(1.3) (see [50, Remark 3.6] for complementary comments).

Next, we establish a version of Theorem 4.2 which enable us to consider locally Lipschitz perturbations for equation (1.2). We have the following result.

Theorem 4.6. Assume that $A$ is sectorial of type $\mu<0$. Let $f:[0, \infty) \times X \rightarrow X$ be a function asymptotically almost periodic in $t$ uniformly in $x \in X$ and assume that there is a continuous and nondecreasing function $L:[0, \infty) \rightarrow[0, \infty)$ such that for each positive number $R$, and $x, y \in X$, $\|x\| \leq R,\|y\| \leq R$, one has

$$
\|f(t, x)-f(t, y)\| \leq L(R)\|x-y\|, \quad \forall t \geq 0,
$$

where $L(0)=0$ and $f(t, 0)=0$ for $t \geq 0$; then there is $\epsilon>0$ such that for each $u_{0}$ with $\left\|u_{0}\right\| \leq \epsilon$ there exists a unique asymptotically almost periodic mild solution of (1.2)-(1.3).

Proof. Let $R>0$ and $0<\lambda<1$ be such that $C M\left(\lambda+|\mu|^{-1 / \alpha} \pi L(R) / \alpha \sin (\pi / \alpha)\right)<1$. We affirm that the assertion holds for $\epsilon=\lambda R$. In fact, we consider $u_{0}$ such that $\left\|u_{0}\right\| \leq \epsilon$. We set

$$
\Phi_{u_{0}}=\left\{u \in A A P(X): u(0)=u_{0},\|u\|_{\infty} \leq R\right\}
$$

endowed with the metric $d(u, v)=\|u-v\|_{\infty}$. We define the operator $\Gamma_{\alpha}$ on the space $\Phi_{u_{0}}$ by (4.3). Let $u \in \Phi_{u_{0}}$; we next show that $\Gamma_{\alpha} u \in \Phi_{u_{0}}$. We have the estimate $\left\|\Gamma_{\alpha} u(t)\right\| \leq C M(\lambda+$ $\left.|\mu|^{-1 / \alpha} \pi L(R) / \alpha \sin (\pi / \alpha)\right) R \leq R$, that is, $\Gamma_{\alpha}\left(\Phi_{u_{0}}\right) \subset \Phi_{u_{0}}$.

On the other hand, for $u_{1}, u_{2} \in \Phi_{u_{0}}$ we obtain

$$
\begin{aligned}
\left\|\Gamma_{\alpha} u_{1}(t)-\Gamma_{\alpha} u_{2}(t)\right\| & \leq C M \int_{0}^{t} \frac{L(R)\left\|u_{1}(s)-u_{2}(s)\right\|}{1+|\mu|(t-s)^{\alpha}} d s \\
& \leq \frac{C M|\mu|^{-1 / \alpha} \pi L(R)}{\alpha \sin (\pi / \alpha)}\left\|u_{1}-u_{2}\right\|_{\infty} \\
& \leq(1-C M \lambda)\left\|u_{1}-u_{2}\right\|_{\infty} .
\end{aligned}
$$

To conclude, we note that $1-C M \lambda<1$, which means that $\Gamma_{\alpha}$ is a $(1-C M \lambda)$-contraction. This completes the proof.

Theorem 4.7. Assume that $A$ is sectorial of type $\mu<0$. Let $f:[0, \infty) \times X \rightarrow X$ be an asymptotically almost periodic in $t$ uniformly in $x \in X$ that satisfies the following conditions.

$\left(H^{*} 1\right)$ There is a continuous nondecreasing function $W:[0, \infty) \rightarrow[0, \infty)$ such that $\|f(t, x)\| \leq W(\|x\|)$ for all $t \geq 0$ and $x \in X$.

$\left(H^{*} 2\right) f(t, x)$ is uniformly continuous on bounded sets of $X$ uniformly in $t \geq 0$. 
$\left(H^{*} 3\right)$ For each $v \geq 0, \lim _{t \rightarrow \infty}\left(1 /\left(h^{*}(t)\right)\right) \int_{0}^{t}\left(W\left(v h^{*}(s)\right) /\left(1+|\mu|(t-s)^{\alpha}\right)\right) d s=0$, where $h^{*}$ is given by Lemma 2.16. Set

$$
\beta^{*}(v):=\sup _{t \geq 0} \frac{1}{h^{*}(t)}\left(\left\|S_{\alpha}(t) u_{0}\right\|+C M \int_{0}^{t} \frac{W\left(v h^{*}(s)\right)}{1+|\mu|(t-s)^{\alpha}} d s\right)
$$

where $C$ and $M$ are constants given in (2.1).

$\left(H^{*} 4\right)$ For each $\epsilon>0$ there is $\delta>0$ such that, for every $u, v \in C_{h^{*}}(X),\|u-v\|_{h^{*}} \leq \delta$ implies that

$$
\sup _{t \geq 0} \int_{0}^{t} \frac{\|f(s, v(s))-f(s, u(s))\|}{1+|\mu|(t-s)^{\alpha}} d s \leq \epsilon
$$

for all $t \in \mathbb{R}$.

$\left(H^{*} 5\right)$ For all $0 \leq a<b$, and $r>0$, the set $\left\{f\left(s, h^{*}(s) x\right): a \leq s \leq b, x \in X,\|x\| \leq r\right\}$ is relatively compact in $X$.

$\left(H^{*} 6\right) \liminf \xi \rightarrow \infty \xi /\left(\beta^{*}(\xi)\right)>1$.

Then problem (1.2)-(1.3) has an asymptotically almost periodic mild solution.

Proof. We define the operator $\Gamma_{\alpha}$ on $C_{h^{*}}(X)$ as in (4.3). We show that $\Gamma_{\alpha}$ has a fixed point in $A A P(X)$.

(i) For $u \in C_{h^{*}}(X)$, we have that

$$
\frac{\left\|\Gamma_{\alpha} u(t)\right\|}{h^{*}(t)} \leq \frac{C M\left\|u_{0}\right\|}{h^{*}(t)}+\frac{C M}{h^{*}(t)} \int_{0}^{t} \frac{W\left(\|u\|_{h^{*}} h^{*}(s)\right)}{1+|\mu|(t-s)^{\alpha}} d s .
$$

It follows from $\left(\mathrm{H}^{*} 3\right)$ that $\Gamma_{\alpha}: C_{h^{*}}(X) \rightarrow C_{h^{*}}(X)$. From condition $\left(\mathrm{H}^{*} 4\right)$ it follows that $\Gamma_{\alpha}$ is a continuous map.

(ii) We next show that $\Gamma_{\alpha}$ is completely continuous. Let $V=\Gamma_{\alpha}\left(B_{r}\left(C_{h^{*}}(X)\right)\right)$ and $v=\Gamma_{\alpha}(u)$ for $u \in B_{r}\left(C_{h^{*}}(X)\right)$. Initially, we can infer that $V_{b}(t)$ is a relatively compact subset of $X$ for each $t \in[0, b]$. In fact, using condition $\left(\mathrm{H}^{*} 5\right)$ we get that $K=\left\{S_{\alpha}(s) f(\xi, h(\xi) x): 0 \leq s \leq t, 0 \leq \xi \leq t,\|x\| \leq r\right\}$ is relatively compact. It is easy to see that $V_{b}(t) \subset S_{\alpha}(t) u_{0}+t \overline{C(K)}$, which establishes our assertion. From the decomposition of $v(t+s)-v(t)$ given by $\left(S_{\alpha}(t+s)-S_{\alpha}(t)\right) u_{0}+\int_{t}^{t+s} S_{\alpha}(t+s-$ $\xi) f(\xi, u(\xi)) d \xi+\int_{0}^{t}\left(S_{\alpha}(\xi+s)-S_{\alpha}(\xi)\right) f(t-\xi, u(t-\xi)) d \xi$, it follows that the set $V_{b}$ is equicontinuous. We can show that $\lim _{t \rightarrow \infty}\|v(t)\| / h^{*}(t)=0$ uniformly for all $u \in B_{r}\left(C_{h^{*}}(X)\right)$. From Lemma 2.16, we deduce that $V$ is relatively compact set in $C_{h^{*}}(X)$.

We note that the set $\left\{u^{\lambda}: u^{\lambda}=\lambda \Gamma_{\alpha}\left(u^{\lambda}\right), \lambda \in(0,1)\right\}$ is bounded. In fact, it follows from condition $\left(\mathrm{H}^{*} 6\right)$ and the estimate $\left\|u^{\lambda}\right\|_{h^{*}} \leq \beta^{*}\left(\left\|u^{\lambda}\right\|_{h^{*}}\right)$. It follows, from Lemmas 2.9 and 2.13 , that $\Gamma_{\alpha}(A A P(X)) \subset A A P(X)$. The remaining of proof makes use of a similar argument already done in the proof of Theorem 3.5. 


\section{Asymptotically Almost Automorphic Solutions of Fractional Integro Differential Neutral Equations}

This section is mainly concerned with the existence and uniqueness of an asymptotically almost automorphic mild solution to the fractional integro differential neutral equation

$$
\begin{gathered}
\frac{d}{d t}(u(t)+g(t, u(t)))=\int_{0}^{t} \frac{(t-s)^{\alpha-2}}{\Gamma(\alpha-1)} A(u(s)+g(s, u(s)) d s+f(t, u(t))), \quad t \geq 0, \\
u(0)=u_{0} \in X,
\end{gathered}
$$

where $1<\alpha<2, A$ is a linear densely defined operator of sectorial type, and $f, g$ are functions subject to some additional conditions.

Definition 5.1. Suppose that $A$ generates an integrable solution operator $S_{\alpha}(t)$. A function $u:[0, \infty) \rightarrow X$ satisfying the integral equation

$$
u(t)=S_{\alpha}(t)\left(u_{0}+g\left(0, u_{0}\right)\right)-g(t, u(t))+\int_{0}^{t} S_{\alpha}(t-s) f(s, u(s)) d s, \quad t \geq 0
$$

is called a mild solution of problem (5.1).

We have the following result.

Theorem 5.2. Assume that $A$ is sectorial of type $\mu<0$. Let $f, g:[0, \infty) \times X \rightarrow X$ be two functions asymptotically almost automorphic in $t$ uniformly for $x$ in compact subsets of $X$ such that

$$
\begin{array}{ll}
\|f(t, x)-f(t, y)\| \leq L_{f}\|x-y\|, \quad \forall x, y \in X, \quad \forall t \geq 0, \\
\|g(t, x)-g(t, y)\| \leq L_{g}\|x-y\|, \quad \forall x, y \in X, \quad \forall t \geq 0 .
\end{array}
$$

If $L_{g}+\left(\left(C M|\mu|^{-1 / \alpha} \pi L_{f}\right) / \alpha \sin (\pi / \alpha)\right)<1$, where $C$ and $M$ are the constants given in (2.1), then problem (5.1) has a unique asymptotically almost automorphic mild solution.

Proof. We define the operator $\Upsilon_{\alpha}$ on the space $A A A([0, \infty), X)$ by

$$
\Upsilon_{\alpha} u(t)=S_{\alpha}(t)\left(u_{0}+g\left(0, u_{0}\right)\right)-g(t, u(t))+\int_{0}^{t} S_{\alpha}(t-s) f(s, u(s)) d s
$$

Applying Lemma 2.22, we infer that $g(\cdot, u(\cdot))$ and $f(\cdot, u(\cdot))$ belong to $A A A([0, \infty), X)$. By Lemma 2.21, we obtain that $\Upsilon_{\alpha}$ is $A A A([0, \infty), X)$-valued. Furthermore, we have the estimate

$$
\begin{aligned}
\left\|\Upsilon_{\alpha} u(t)-\Upsilon_{\alpha} v(t)\right\| & \leq L_{g}\|u(t)-v(t)\|+C M L_{f} \int_{0}^{t} \frac{\|u(s)-v(s)\|}{1+|\mu|(t-s)^{\alpha}} d s \\
& \leq\left(L_{g}+\frac{C M L_{f}|\mu|^{-1 / \alpha} \pi}{\alpha \sin (\pi / \alpha)}\right)\|u-v\|_{\infty}
\end{aligned}
$$


which proves that $\Upsilon_{\alpha}$ is a contraction we conclude that $\Upsilon_{\alpha}$ has a unique fixed point in $A A A([0, \infty), X)$. This completes the proof.

Next, we establish a local version of the previous result.

Theorem 5.3. Assume that $A$ is sectorial of type $\mu<0$. Let $f, g:[0, \infty) \times X \rightarrow X$ be two functions asymptotically almost automorphic in $t$ uniformly for $x$ in compact subsets of $X$ and assume that there are continuous and nondecreasing functions $L_{f}, L_{g}:[0, \infty) \rightarrow[0, \infty)$ such that for each positive number $R$, and $x, y \in X,\|x\| \leq R,\|y\| \leq R$, one has

$$
\|f(t, x)-f(t, y)\| \leq L_{f}(R)\|x-y\|,\|g(t, x)-g(t, y)\| \leq L_{g}(R)\|x-y\|,
$$

for all $t \geq 0$, where $L_{f}(0)=L_{g}(0)=0$ and $f(t, 0)=g(t, 0)=0$ for every $t \geq 0$. Then there is $\epsilon>0$ such that $u_{0}$ satisfies $\left\|u_{0}\right\| \leq \epsilon$; then there is a unique asymptotically almost automorphic mild solution of (5.1).

Proof. Let $R>0$ and $\lambda \in(0,1)$ be such that

$$
H:=C M\left(1+L_{g}(\lambda R)\right) \lambda+L_{g}(R)+\frac{C M L_{f}(R)|\mu|^{-1 / \alpha} \pi}{\alpha \sin (\pi / \alpha)}<1,
$$

where $C$ and $M$ are the constants given in (2.1). We consider $u_{0}$ such that $\left\|u_{0}\right\| \leq \epsilon$, with $\epsilon=\lambda R$; we define the space $\Phi_{u_{0}}=\left\{u \in A A A([0, \infty), X): u(0)=u_{0},\|u\|_{\infty} \leq R\right\}$ endowed with the metric $d(u, v)=\|u-v\|_{\infty}$. We also define the operator $\Upsilon_{\alpha}$ on the space $\Phi_{u_{0}}$ by (5.4). Let $u$ be in $\Phi_{u_{0}}$ in a similar way as that of proof of Theorem 5.2; we have that $\Upsilon_{\alpha} u \in A A A([0, \infty), X)$. Moreover, we obtain the estimate

$$
\left\|\Upsilon_{\alpha} u(t)\right\| \leq C M\left(1+L_{g}(\lambda R)\right) \lambda R+L_{g}(R) R+\frac{C M L_{f}(R) R|\mu|^{-1 / \alpha} \pi}{\alpha \sin (\pi / \alpha)}=H R<R .
$$

Therefore $\Upsilon_{\alpha}\left(\boldsymbol{\Phi}_{u_{0}}\right) \subset \boldsymbol{\Phi}_{u_{0}}$. On the other hand, for $u, v \in \Phi_{u_{0}}$, we see that

$$
\left\|\Upsilon_{\alpha} u-\Upsilon_{\alpha} v\right\|_{\infty} \leq\left(L_{g}(R)+\frac{C M L_{f}(R)|\mu|^{-1 / \alpha} \pi}{\alpha \sin (\pi / \alpha)}\right)\|u-v\|_{\infty}
$$

which shows that $\Upsilon_{\alpha}$ is a contraction from $\Phi_{u_{0}}$ into $\Phi_{u_{0}}$. The assertion is now a consequence of the contraction mapping principle.

Remark 5.4. A similar result was obtained by Diagana et al. [76] for the existence of asymptotically almost automorphic solutions to some abstract partial neutral integrodifferential equations.

Theorem 5.5. Assume that $A$ is sectorial of type $\mu<0$ and that conditions $\left(H^{*} 1\right),\left(H^{*} 3\right),\left(H^{*} 4\right)$ and $\left(H^{*} 5\right)$ hold. In addition, suppose that the following properties hold. 
(A1) The functions $f, g:[0, \infty) \times X \rightarrow X$ are asymptotically almost automorphic in $t$ and uniformly for $x$ in compact subsets of $X$ and uniformly continuous on bounded sets of $X$ uniformly in $t \geq 0$.

(A2) There is a constant $L_{g}>0$ such that $\left\|g\left(t, h^{*}(t) x\right)-g\left(t, h^{*}(t) y\right)\right\| \leq L_{g}\|x-y\|$ for all $t \geq 0$ and $x, y \in X$ (here $h^{*}$ is given in Lemma 2.16). Set

$$
\Omega(v)=\sup _{t \geq 0} \frac{C M}{h^{*}(t)} \int_{0}^{t} \frac{W\left(v h^{*}(s)\right)}{1+|\mu|(t-s)^{\alpha}} d s
$$

where $C$ and $M$ are the constants given in (2.1).

(A3) $L_{g}+\liminf _{r \rightarrow \infty}(\Omega(r)) / r<1$.

Then problem (5.1) has an asymptotically almost automorphic mild solution.

Proof. We define the operator $\Upsilon_{\alpha}$ on $C_{h^{*}}(X)$ as in (5.4); we consider the decomposition $\Upsilon_{\alpha}=$ $\Upsilon_{\alpha}^{1}+\Upsilon_{\alpha}^{2}$, where

$$
\begin{gathered}
\Upsilon_{\alpha}^{1} u(t)=S_{\alpha}(t)\left(u_{0}+g\left(0, u_{0}\right)\right)-g(t, u(t)), \quad t \geq 0, \\
\Upsilon_{\alpha}^{2} u(t)=\int_{0}^{t} S_{\alpha}(t-s) f(s, u(s)) d s, \quad t \geq 0 .
\end{gathered}
$$

For $u \in C_{h^{*}}(X)$, we have that

$$
\frac{\left\|\Upsilon_{\alpha}^{1} u(t)\right\|}{h^{*}(t)} \leq \frac{1}{h^{*}(t)}\left[C M\left(\left\|u_{0}\right\|+\left\|g\left(0, u_{0}\right)\right\|\right)+L_{g}\|u\|_{h^{*}}+\|g(\cdot, 0)\|_{\infty}\right]
$$

Hence $\Upsilon_{\alpha}^{1}$ is $C_{h^{*}}(X)$-valued. On the other hand, $\Upsilon_{\alpha}^{1}$ is an $L_{g}$-contraction. It follows from the proof of the Theorem 4.7 that $\Upsilon_{\alpha}^{2}$ is completely continuous. From Lemmas 2.21 and 2.22, we have that

$$
\Upsilon_{\alpha}^{i}(A A A([0, \infty), X)) \subset A A A([0, \infty), X), \quad i=1,2
$$

Hence $\Upsilon_{\alpha}(A A A([0, \infty), X)) \subset A A A([0, \infty), X)$ and $\Upsilon_{\alpha}^{2}: A A A([0, \infty), X) \rightarrow A A A([0, \infty), X)$ is completely continuous. Putting $B_{r}:=B_{r}(A A A([0, \infty), X))$, we claim that there is $r>0$ such that $\Upsilon_{\alpha}\left(B_{r}\right) \subset B_{r}$. In fact, if we assume that this assertion is false, then for all $r>0$ we can choose $u^{r} \in B_{r}$ and $t^{r} \geq 0$ such that $\left\|\Upsilon_{\alpha} u^{r}\left(t^{r}\right)\right\| / h^{*}\left(t^{r}\right)>r$. We observe that

$$
\left\|\Upsilon_{\alpha} u^{r}\left(t^{r}\right)\right\| \leq C M\left(\left\|u_{0}\right\|+\left\|g\left(0, u_{0}\right)\right\|\right)+\|g(\cdot, 0)\|_{\infty}+L_{g} r+C M \int_{0}^{t^{r}} \frac{W\left(r h^{*}(s)\right)}{1+|\mu|\left(t^{r}-s\right)^{\alpha}} d s .
$$

Thus $1 \leq L_{g}+\liminf _{r \rightarrow \infty}(\Omega(r) / r)$, which is contrary to assumption (A3). We have that $\Upsilon_{\alpha}^{1}$ is a contraction on $B_{r}$ and $\Upsilon_{\alpha}^{2}\left(B_{r}\right)$ is a compact set. It follows from [77, Corollary 4.3.2] that $\Upsilon_{\alpha}$ has a fixed point $u \in A A A([0, \infty), X)$. More precisely, $u \in A A A([0, \infty), X)$, and this finishes the proof. 


\section{Applications}

To illustrate our results, initially we examine sufficient conditions for the existence and uniqueness of pseudo-almost periodic mild solutions to the fractional relaxation-oscillation equation given by

$$
\begin{gathered}
\partial_{t}^{\alpha} u(t, x)=\partial_{x}^{2} u(t, x)-v u(t, x)+\beta \partial_{t}^{\alpha-1}\left(\left(\sin (t)+\frac{1}{1+t^{2}}\right) \sin (u(t, x))\right), \quad t \in \mathbb{R}, x \in[0, \pi] \\
u(t, 0)=u(t, \pi)=0, \quad t \in \mathbb{R}
\end{gathered}
$$

where $\nu, \beta>0$. To study this system in the abstract form (1.1), we choose the space $X=$ $L^{2}[0, \pi]$ and the operator $A$ defined by $A u=u^{\prime \prime}-v u$, with domain $D(A)=\left\{u \in L^{2}[0, \pi]\right.$ : $\left.u^{\prime \prime} \in L^{2}[0, \pi], u(0)=u(\pi)=0\right\}$. It is well known that $\Delta u=u^{\prime \prime}$ is generator of an analytic semigroup on $L^{2}[0, \pi]$. Hence, $A$ is sectorial of type $\mu=-v<0$. (6.1) can be formulated by the inhomogeneous problem (1.1), where $u(t)(x)=u(t, x)$. Let us consider the nonlinearity $f(t, \phi)(s)=\beta b(t) \sin \phi(s)$ for all $\phi \in X$ and $s \in[0, \pi], t \in \mathbb{R}$ with $b(t)=\sin (t)+\left(1 / 1+t^{2}\right)$, $\beta \in \mathbb{R}$. We observe that $\lim _{r \rightarrow \infty}(1 / 2 r) \int_{-r}^{r}(1 / 1)+t^{2} d t=\lim _{r \rightarrow \infty}(1 / r) \arctan (r)=0$. Hence $b \in P A P(\mathbb{R})$. We observe that $f: \mathbb{R} \times X \rightarrow X$ is pseudo-almost periodic in $t \in \mathbb{R}$, uniformly in $x \in X$ such that (3.6) holds for $L=2|\beta|$. If we assume that $|\beta|<\alpha \sin (\pi / \alpha) /\left(2 C M|v|^{-1 / \alpha} \pi\right)$, then by Corollary 3.4, the fractional relaxation-oscillation equation (6.1) has a unique pseudoalmost periodic mild solution.

Taking $\beta \in(0,1)$ and $\lambda, v>0$, we define the function $F$ as

$$
F(t, u)(x)=e^{-\lambda|t|}\left|\int_{0}^{x} \sin \left(\frac{u(t, \tau)}{\left(\int_{0}^{\pi}|u(t, \xi)|^{2} d \xi\right)^{1 / 2}+1}\right) d \tau\right|^{\beta} \sin (x)
$$

We consider the following fractional relaxation-oscillation equation given by

$$
\begin{gathered}
\partial_{t}^{\alpha} u(t, x)=\partial_{x}^{2} u(t, x)-v u(t, x)+\partial_{t}^{\alpha-1} F(t, u)(x), \quad t \in \mathbb{R}, x \in[0, \pi], \\
u(t, 0)=u(t, \pi)=0, \quad t \in \mathbb{R} .
\end{gathered}
$$

Equation (6.4) can be expressed as an abstract equation of the form (1.1), where

$$
f(t, \phi)(\xi)=e^{-\lambda|t|}\left|\int_{0}^{\xi} \sin \left(\frac{\phi(\tau)}{\|\phi\|_{L^{2}}+1}\right) d \tau\right|^{\beta} \sin (\xi) .
$$

Proposition 6.1. Problem (6.4) has a pseudo-almost periodic mild solution. 
Proof. Let us briefly discuss the proof of this proposition. We get without difficulties the following two estimates:

$$
\begin{gathered}
\|f(t, \phi)\|_{L^{2}} \leq e^{-\lambda|t|} \pi^{(1+\beta) / 2}, \quad t \in \mathbb{R}, \phi \in X, \\
\left\|f\left(t, \phi_{1}\right)-f\left(t, \phi_{2}\right)\right\|_{L^{2}} \leq e^{-\lambda|t|} 3^{\beta} \pi^{(1+\beta) / 2}\left\|\phi_{1}-\phi_{2}\right\|_{L^{2}}^{\beta}, \quad t \in \mathbb{R}, \phi_{1}, \phi_{2} \in X,
\end{gathered}
$$

which are responsible for the fact that $f \in P A P_{0}(\mathbb{R} \times X, X)$ and that $f$ is uniformly continuous on bounded sets of $X$ uniformly in $t \in \mathbb{R}$.

It is straightforward to verify that

$$
\|f(t, \phi)\|_{L^{2}} \leq e^{-\lambda|t|} \pi^{(1+\beta) / 2}\left(\frac{\|\phi\|_{L^{2}}}{\|\phi\|_{L^{2}}+1}\right)^{\beta} \leq \pi^{(1+\beta) / 2}\left(\frac{\|\phi\|_{L^{2}}}{\|\phi\|_{L^{2}}+1}\right)^{\beta}
$$

and $\phi \in X$. Hence, we can define $W$ in $(\mathrm{H} 1)$ by $W(\xi)=\pi^{(1+\beta) / 2}(\xi /(\xi+1))^{\beta}$. Taking $h(t)=e^{\lambda|t|}$, $t \in \mathbb{R} ; u, v \in C_{h}(X)$. From the discussion above, we see that

$$
\begin{gathered}
\frac{1}{h(t)} \int_{-\infty}^{t} \frac{W(v h(s))}{1+|\mu|(t-s)^{\alpha}} d s \leq \frac{v^{\beta} \pi^{(3+\beta) / 2}|\mu|^{-1 / \alpha}}{\alpha \sin (\pi / \alpha)} \frac{1}{h(t)} \longrightarrow 0, \quad \text { as }|t| \longrightarrow \infty, \\
\sup _{t \in \mathbb{R}} \int_{-\infty}^{t} \frac{\|f(s, u(s))-f(s, v(s))\|_{L^{2}}}{1+|\mu|(t-s)^{\alpha}} d s \leq \frac{3^{\beta} \pi^{(3+\beta) / 2}|\mu|^{-1 / \alpha}}{\alpha \sin (\pi / \alpha)}\|u-v\|_{h^{\prime}}^{\beta}
\end{gathered}
$$

which means that conditions $(\mathrm{H} 3)$ and $(\mathrm{H} 4)$ of Theorem 3.5 are satisfied. An easy computation leads to $\liminf _{\xi \rightarrow \infty}(\xi / \beta(\xi))>1$. An argument involving Simon's theorem (see [78, Theorem 1, pages 71-74]) proves that the set $K=\left\{f\left(s, e^{\lambda|s|} \phi\right): a \leq s \leq\right.$ $\left.b, \phi \in L^{2}[0, \pi],\|\phi\|_{L^{2}} \leq r\right\}$ is relatively compact in $L^{2}[0, \pi]$. In fact, we can verify that $\left\|f\left(s, e^{\lambda|s|} \phi\right)\right\|_{L^{2}} \leq \pi^{(1+\beta) / 2}, \phi \in L^{2}[0, \pi]$. Hence, for $a_{1}<a_{2}, \int_{a_{1}}^{a_{2}} f\left(s, e^{\lambda|s|} \phi\right)(\xi) d \xi$ is bounded uniformly in $s$ and $\phi$. On the other hand, we can infer the following estimate:

$$
\left|f\left(s, e^{\lambda|s|} \phi\right)(\xi)-f\left(s, e^{\lambda|s|} \phi\right)\left(\xi^{\prime}\right)\right| \leq\left|\xi-\xi^{\prime}\right|^{\beta}+\pi^{\beta}\left|\xi-\xi^{\prime}\right|
$$

Therefore,

$$
\int_{0}^{\pi-h}\left|f\left(s, e^{\lambda|s|} \phi\right)(\xi+h)-f\left(s, e^{\lambda|s|} \phi\right)(\xi)\right|^{2} d \xi \longrightarrow 0 \quad \text { as } h \longrightarrow 0
$$

uniformly in $s$ and $\phi$. Finally, Simon's theorem leads to the conclusion that $K$ is relatively compact. Using Theorem 3.5, equation (6.4) has a pseudo-almost periodic mild solution. 
Next, we examine the existence and uniqueness of an asymptotically almost automorphic mild solution to the fractional differential equation

$$
\begin{gathered}
\frac{\partial}{\partial t} u(t, \xi)=J_{t}^{\alpha-1}\left(\frac{\partial^{2}}{\partial \xi^{2}}-v\right) u(t, \xi)+a(t) f(u(t, \xi)), \quad t \geq 0, \xi \in[0, \pi], \\
u(t, 0)=u(t, \pi)=0, \quad t \geq 0, \\
u(0, \xi)=u_{0}(\xi), \quad \xi \in[0, \pi]
\end{gathered}
$$

where $u_{0} \in L^{2}[0, \pi], a, f$ are appropriate functions, and $\int_{t}^{\alpha-1} g(t)=\int_{0}^{t}\left((t-s)^{\alpha-2} / \Gamma(\alpha-\right.$ 1)) $g(s) d s$. From Corollary 4.3 , we can deduce the following result.

Proposition 6.2. Assume that $a(\cdot)$ is an asymptotically almost periodic function and that there exists a constant $L_{f}>0$ such that $|f(x)-f(y)| \leq L_{f}|x-y|$ for all $x, y \in \mathbb{R}$. If $\|a\|_{\infty} \leq$ $\left(\alpha \sin (\pi / \alpha) / C M|v|^{-1 / \alpha} \pi L_{f}\right)$, then (6.11) has a unique asymptotically almost periodic mild solution.

We consider the fractional differential equation

$$
\begin{gathered}
\frac{\partial}{\partial t} u(t, \xi)=J_{t}^{\alpha-1}\left(\frac{\partial^{2}}{\partial \xi^{2}}-v\right) u(t, \xi)+a(t)\left(\int_{0}^{\xi} u(t, \tau) d \tau\right)^{2}, \quad t \geq 0, \xi \in[0, \pi], \\
u(t, 0)=u(t, \pi)=0, \quad t \geq 0, \\
u(0, \xi)=u_{0}(\xi), \quad \xi \in[0, \pi] .
\end{gathered}
$$

From Theorem 4.6, we deduce the following result.

Proposition 6.3. Assume that $a(\cdot)$ is an asymptotically almost periodic function, then there is $\epsilon>0$ such that for each $u_{0}$ with $\left\|u_{0}\right\|_{L^{2}} \leq \epsilon$ there exists a unique asymptotically almost periodic mild solution of (6.12)-(6.14).

Proof. The proof is straightforward. Indeed, (6.12) can be expressed as an abstract equation of form (1.2), where $f(t, \phi)(\xi)=a(t)\left(\int_{0}^{\xi} \phi(\tau) d \tau\right)^{2}, t \geq 0, \phi \in L^{2}[0, \pi]$. We observe that $\| f\left(t, \phi_{1}\right)-$ $f\left(t, \phi_{2}\right)\left\|_{L^{2}} \leq\right\| a\left\|_{\infty} \pi^{3 / 2}\left(\left\|\phi_{1}\right\|_{L^{2}}+\left\|\phi_{2}\right\|_{L^{2}}\right)\right\| \phi_{1}-\phi_{2} \|_{L^{2}}$, for all $t \geq 0$ and $\phi_{1}, \phi_{2} \in L^{2}[0, \pi]$. Hence the perturbation is locally Lipschitz. We remark that $f$ is asymptotically almost periodic in $t$ uniformly in $\phi \in L^{2}[0, \pi]$, as we mentioned before, by using Theorem 4.6.

Take $\beta \in(0,1)$ and $\lambda, v>0$. We define the function $F$ by

$$
F(t, u)(x)=e^{-\lambda t}\left|\int_{0}^{x} u(t, \tau) d \tau\right|^{\beta} \sin (x)
$$


We examine asymptotically almost periodic mild solution to the fractional relaxationoscillation equation given by

$$
\begin{gathered}
\partial_{t}^{\alpha} u(t, x)=\partial_{t}^{2} u(t, x)-v u(t, x)+\partial_{t}^{\alpha-1} F(t, u)(x), \quad t \geq 0, x \in[0, \pi] \\
u(t, 0)=u(t, \pi)=0, \quad t \geq 0 \\
u(t, \xi)=u_{0}(\xi), \quad \xi \in[0, \pi]
\end{gathered}
$$

where $u_{0} \in L^{2}[0, \pi]$.

Proposition 6.4. Problem (6.16) has an asymptotically almost periodic mild solution.

Proof. We briefly recall some argument of the proof. Problem (6.16) can be written as an abstract problem of the form (1.2)-(1.3) in $X=L^{2}[0, \pi]$, where the perturbation associated is

$$
f(t, \phi)(\xi)=e^{-\lambda t}\left|\int_{0}^{\xi} \phi(\tau) d \tau\right|^{\beta} \sin (\xi), \quad \xi \in[0, \pi] .
$$

We can choose the function $W$ in $\left(\mathrm{H}^{*} 1\right)$ by $W(\xi)=\pi^{(1+\beta) / 2} \xi^{\beta}$. From the estimate

$$
\left\|f\left(t, \phi_{1}\right)-f\left(t, \phi_{2}\right)\right\|_{L^{2}} \leq e^{-\lambda t}{\pi^{(1+\beta) / 2}}\left\|\phi_{1}-\phi_{2}\right\|_{L^{2}}^{\beta}, \quad t \geq 0, \phi \in L^{2}[0, \pi],
$$

we get conditions $\left(\mathrm{H}^{*} 2\right)$ and $\left(\mathrm{H}^{*} 4\right)$, the latter being considered with $h^{*}(t)=e^{\lambda t}, t \geq 0$.

We can infer that

$$
\frac{1}{e^{\lambda t}} \int_{0}^{t} \frac{W\left(v e^{\lambda s}\right)}{1+|\mu|(t-s)^{\alpha}} d s \leq \frac{1}{e^{\lambda(1-\beta) t}} \frac{v^{\beta} \pi^{(3+\beta) / 2}|\mu|^{-1 / \alpha}}{\alpha \sin (\pi / \alpha)} \longrightarrow 0, \quad \text { as } t \longrightarrow \infty .
$$

Hence condition $\left(\mathrm{H}^{*} 3\right)$ is fulfilled. By looking at the estimates

$$
\begin{gathered}
\left\|f\left(s, e^{\lambda s} \phi\right)\right\|_{L^{2}} \leq \pi^{(1+\beta) / 2} r^{\beta}, \quad \phi \in L^{2}[0, \pi],\|\phi\|_{L^{2}} \leq r, \\
\left\|f\left(s, e^{\lambda s} \phi\right)(\xi)-f\left(s, e^{\lambda s} \phi\right)\left(\xi^{\prime}\right)\right\| \leq r^{\beta}\left|\xi-\xi^{\prime}\right|^{\beta / 2}+\pi^{\beta / 2} r^{\beta}\left|\xi-\xi^{\prime}\right|
\end{gathered}
$$

and using Simon's theorem, we conclude that condition $\left(\mathrm{H}^{*} 5\right)$ holds. Consequently, by Theorem 4.7 we can assert that problem (6.16) has an asymptotically almost periodic mild solution. This completes the proof of Proposition 6.4.

Remark 6.5. It is easy to check that results in Section 5 are applicable to similar fractional differential equations as those treated in this section. For the sake of shortness, the details are left to the reader. 


\section{Acknowledgment}

Claudio Cuevas is partially supported by CNPQ/Brazil under Grant no. 300365/2008-0.

\section{References}

[1] V. V. Anh and R. Mcvinish, "Fractional differential equations driven by Lévy noise," Journal of Applied Mathematics and Stochastic Analysis, vol. 16, no. 2, pp. 97-119, 2003.

[2] R. Gorenflo and F. Mainardi, "Fractional calculus: integral and differential equations of fractional order," in Fractals and Fractional Calculus in Continuum Mechanics, A. Carpinteri and F. Mainardi, Eds., vol. 378 of CISM Courses and Lectures, pp. 223-276, Springer, Vienna, Austria, 1997.

[3] R. Hilfer, Ed., Applications of Fractional Calculus in Physics, World Scientific, River Edge, NJ, USA, 2000.

[4] T. Hu and Y. Wang, "Numerical detection of the lowest "efficient dimensions" for chaotic fractional differential systems," Open Mathematics Journal, vol. 1, pp. 11-18, 2008.

[5] A. A. Kilbas, H. M. Srivastava, and J. J. Trujillo, Theory and Applications of Fractional Differential Equations, vol. 204 of North-Holland Mathematics Studies, Elsevier Science B.V., Amsterdam, The Netherlands, 2006.

[6] K. S. Miller and B. Ross, An Introduction to the Fractional Calculus and Fractional Differential Equations, John Wiley \& Sons, New York, NY, USA, 1993.

[7] I. Podlubny, Fractional Differential Equations. An Introduction to Fractional Derivatives, Fractional Differential Equations, to Methods of Their Solution and some of Their Application, vol. 198 of Mathematics in Science and Engineering, Academic Press, San Diego, Calif, USA, 1999.

[8] S. G. Samko, A. A. Kilbas, and O. I. Marichev, Fractional Integrals and Derivatives. Theory and Applications, Gordon and Breach Science, Yverdon, France, 1993.

[9] K. Diethelm and A. D. Freed, "On the solution of nonlinear fractional order equations used in the modeling of viscoplasticity," in Scientific Computing in Chemical Engineering II-Computational Fluid Dynamics, Reaction Engineering and Molecular Properties, F. Keil, W. Mackens, H. Voss, and J. Werther, Eds., pp. 217-224, Springer, Heidelberg, Germany, 1999.

[10] S. D. Eidelman and A. N. Kochubei, "Cauchy problem for fractional diffusion equations," Journal of Differential Equations, vol. 199, no. 2, pp. 211-255, 2004.

[11] W. Lin, "Global existence theory and chaos control of fractional differential equations," Journal of Mathematical Analysis and Applications, vol. 332, no. 1, pp. 709-726, 2007.

[12] A. Oustalup, Systéms Asservis D'ordre Fractionaire, Éditions Masson, 1983.

[13] A. Oustalup, La Dérivation non Entière: Théorie, Synthèse, Applications, Série Automatique, Editions Hermès, 1995.

[14] I. Podlubny, I. Petráš, B. M. Vinagre, P. O’Leary, and L. Dorčák, “Analogue realizations of fractionalorder controllers. Fractional order calculus and its applications," Nonlinear Dynamics, vol. 29, no. 1-4, pp. 281-296, 2002.

[15] B. Ross, Ed., Fractional Calculus and Its Applications, Lecture Notes in Mathematics, vol. 457, Springer, Berlin, Germany, 1975.

[16] M. Benchohra, J. Henderson, S. K. Ntouyas, and A. Ouahab, "Existence results for fractional order functional differential equations with infinite delay," Journal of Mathematical Analysis and Applications, vol. 338, no. 2, pp. 1340-1350, 2008.

[17] R. P. Agarwal, M. Benchohra, and S. Hamani, "A survey on existence results for boundary value problems of nonlinear fractional differential equations and inclusions," to appear in Acta Applicandae Mathematicae.

[18] R. P. Agarwal, M. Benchohra, and S. Hamani, "Boundary value problems for fractional differential equations," Georgian Mathematical Journal, vol. 16, no. 3, pp. 401-411, 2009.

[19] R. P. Agarwal, M. Belmekki, and M. Benchohra, "A survey on semilinear differential equations and inclusions involving Riemann-Liouville fractional derivative," Advances in Difference Equations, vol. 2009, Article ID 981728, 47 pages, 2009.

[20] V. Lakshmikantham, "Theory of fractional functional differential equations," Nonlinear Analysis: Theory, Methods E Applications, vol. 69, no. 10, pp. 3337-3343, 2008.

[21] V. Lakshmikantham and A. S. Vatsala, "Basic theory of fractional differential equations," Nonlinear Analysis: Theory, Methods \& Applications, vol. 69, no. 8, pp. 2677-2682, 2008.

[22] V. Lakshmikantham and A. S. Vatsala, "Theory of fractional differential inequalities and applications," Communications in Applied Analysis, vol. 11, no. 3-4, pp. 395-402, 2007. 
[23] G. M. Mophou and G. M. N'Guérékata, "Mild solutions for semilinear fractional differential equations," Electronic Journal of Differential Equations, no. 21, pp. 1-9, 2009.

[24] M. M. El-Borai, "Some probability densities and fundamental solutions of fractional evolution equations," Chaos, Solitons and Fractals, vol. 14, no. 3, pp. 433-440, 2002.

[25] M. M. El-Borai, "Semigroups and some nonlinear fractional differential equations," Applied Mathematics and Computation, vol. 149, no. 3, pp. 823-831, 2004.

[26] M. M. El-Borai, "The fundamental solutions for fractional evolution equations of parabolic type," Journal of Applied Mathematics and Stochastic Analysis, no. 3, pp. 197-211, 2004.

[27] G. M. Mophou, O. Nakoulima, and G. M. N'Guérékata, “Existence results for some fractional differential equations with nonlocal conditions," submitted.

[28] G. M. Mophou and G. M. N'Guérékata, "Existence of the mild solution for some fractional differential equations with nonlocal conditions," Semigroup Forum, vol. 79, no. 2, pp. 315-322, 2009.

[29] G. M. N'Guérékata, "A Cauchy problem for some fractional abstract differential equation with non local conditions," Nonlinear Analysis: Theory, Methods \& Applications, vol. 70, no. 5, pp. 1873-1876, 2009.

[30] A. M. A. El-Sayed and A.-G. Ibrahim, "Multivalued fractional differential equations," Applied Mathematics and Computation, vol. 68, no. 1, pp. 15-25, 1995.

[31] M. Benchohra, J. Henderson, S. K. Ntouyas, and A. Ouahab, "Existence results for fractional functional differential inclusions with infinite delay and applications to control theory," Fractional Calculus \& Applied Analysis, vol. 11, no. 1, pp. 35-56, 2008.

[32] J. Henderson and A. Ouahab, "Fractional functional differential inclusions with finite delay," Nonlinear Analysis: Theory, Methods \& Applications, vol. 70, no. 5, pp. 2091-2105, 2009.

[33] A. Ouahab, "Some results for fractional boundary value problem of differential inclusions," Nonlinear Analysis: Theory, Methods \& Applications, vol. 69, no. 11, pp. 3877-3896, 2008.

[34] C. Cuevas and C. Lizama, "Almost automorphic solutions to a class of semilinear fractional differential equations," Applied Mathematics Letters, vol. 21, no. 12, pp. 1315-1319, 2008.

[35] C. Cuevas and C. Lizama, "Almost automorphic solutions to integral equations on the line," Semigroup Forum, vol. 79, no. 3, pp. 461-472, 2009.

[36] C. Y. Zhang, "Pseudo-almost-periodic solutions of some differential equations," Journal of Mathematical Analysis and Applications, vol. 181, no. 1, pp. 62-76, 1994.

[37] C. Y. Zhang, "Integration of vector-valued pseudo-almost periodic functions," Proceedings of the American Mathematical Society, vol. 121, no. 1, pp. 167-174, 1994.

[38] C. Y. Zhang, "Pseudo almost periodic solutions of some differential equations. II," Journal of Mathematical Analysis and Applications, vol. 192, no. 2, pp. 543-561, 1995.

[39] C. Zhang, Almost Periodic Type Functions and Ergodicity, Science Press, Beijing, China, 2003.

[40] E. Ait Dads and O. Arino, "Exponential dichotomy and existence of pseudo almost-periodic solutions of some differential equations," Nonlinear Analysis: Theory, Methods E Applications, vol. 27, no. 4, pp. 369-386, 1996.

[41] E. Ait Dads, K. Ezzinbi, and O. Arino, "Pseudo almost periodic solutions for some differential equations in a Banach space," Nonlinear Analysis: Theory, Methods E Applications, vol. 28, no. 7, pp. 1141-1155, 1997.

[42] B. Amir and L. Maniar, "Composition of pseudo-almost periodic functions and Cauchy problems with operator of nondense domain," Annales Mathématiques Blaise Pascal, vol. 6, no. 1, pp. 1-11, 1999.

[43] C. Cuevas and E. Hernández M., "Pseudo-almost periodic solutions for abstract partial functional differential equations," Applied Mathematics Letters, vol. 22, no. 4, pp. 534-538, 2009.

[44] C. Cuevas and M. Pinto, "Existence and uniqueness of pseudo almost periodic solutions of semilinear Cauchy problems with non dense domain," Nonlinear Analysis: Theory, Methods E Applications, vol. 45, no. 1, pp. 73-83, 2001.

[45] T. Diagana, "Pseudo almost periodic solutions to some differential equations," Nonlinear Analysis: Theory, Methods \& Applications, vol. 60, no. 7, pp. 1277-1286, 2005.

[46] T. Diagana, C. M. Mahop, and G. M. N'Guérékata, "Pseudo-almost-periodic solutions to some semilinear differential equations," Mathematical and Computer Modelling, vol. 43, no. 1-2, pp. 89-96, 2006.

[47] T. Diagana, C. M. Mahop, G. M. N'Guérékata, and B. Toni, “Existence and uniqueness of pseudoalmost periodic solutions to some classes of semilinear differential equations and applications," Nonlinear Analysis: Theory, Methods \& Applications, vol. 64, no. 11, pp. 2442-2453, 2006.

[48] T. Diagana and C. M. Mahop, "Pseudo almost periodic solutions to a neutral delay integral equation," Cubo, vol. 9, no. 1, pp. 47-55, 2007. 
[49] T. Diagana, "Existence of pseudo almost periodic solutions to some classes of partial hyperbolic evolution equations," Electronic Journal of Qualitative Theory of Differential Equations, no. 3, p. 12, 2007.

[50] C. Cuevas and J. C. de Souza, "S-asymptotically $\omega$-periodic solutions of semilinear fractional integrodifferential equations," Applied Mathematics Letters, vol. 22, no. 6, pp. 865-870, 2009.

[51] C. Cuevas and J. C. de Souza, "Existence of $S$-asymptotically $\omega$-periodic solutions for fractional order functional integro-differential equations with infinite delay," Nonlinear Analysis: Theory, Methods $\mathcal{E}$ Applications, vol. 72, no. 3-4, pp. 1683-1689, 2010.

[52] M. Haase, The Functional Calculus for Sectorial Operators, vol. 169 of Operator Theory: Advances and Applications, Birkhäuser, Basel, Switzerland, 2006.

[53] E. Cuesta, "Asymptotic behaviour of the solutions of fractional integro-differential equations and some time discretizations," Discrete and Continuous Dynamical Systems, pp. 277-285, 2007.

[54] J. Prüss, Evolutionary Integral Equations and Applications, vol. 87 of Monographs in Mathematics, Birkhäuser, Basel, Switzerland, 1993.

[55] G. Gripenberg, S.-O. Londen, and O. Staffans, Volterra Integral and Functional Equations, vol. 34 of Encyclopedia of Mathematics and Its Applications, Cambridge University Press, Cambridge, UK, 1990.

[56] W. Arendt, C. J. K. Batty, M. Hieber, and F. Neubrander, Vector-Valued Laplace Transforms and Cauchy Problems, vol. 96 of Monographs in Mathematics, Birkhäuser, Basel, Switzerland, 2001.

[57] O. Fattorini, Second Order Differential Equations in Banach Spaces, vol. 108 of North-Holland Mathematics Studies, North-Holland, Amsterdam, The Netherlands, 1985.

[58] C. Lizama, "On approximation and representation of $k$-regularized resolvent families," Integral Equations and Operator Theory, vol. 41, no. 2, pp. 223-229, 2001.

[59] C. Lizama and H. Prado, "Rates of approximation and ergodic limits of regularized operator families," Journal of Approximation Theory, vol. 122, no. 1, pp. 42-61, 2003.

[60] C. Lizama and J. Sánchez, "On perturbation of $k$-regularized resolvent families," Taiwanese Journal of Mathematics, vol. 7, no. 2, pp. 217-227, 2003.

[61] S.-Y. Shaw and J.-C. Chen, "Asymptotic behavior of $(a, k)$-regularized resolvent families at zero," Taiwanese Journal of Mathematics, vol. 10, no. 2, pp. 531-542, 2006.

[62] S. Zaidman, Almost-Periodic Functions in Abstract Spaces, vol. 126 of Research Notes in Mathematics, Pitman, Boston, Mass, USA, 1985.

[63] A. M. Fink, Almost Periodic Differential Equations, Lecture Notes in Mathematics, vol. 377, Springer, Berlin, Germany, 1974.

[64] C. Zhang, Pseudo almost periodic functions and their applications, thesis, The University of Western Ontario, 1992.

[65] H.-X. Li, F.-L. Huang, and J.-Y. Li, “Composition of pseudo almost-periodic functions and semilinear differential equations," Journal of Mathematical Analysis and Applications, vol. 255, no. 2, pp. 436-446, 2001.

[66] H. R. Henríquez and C. Lizama, "Compact almost automorphic solutions to integral equations with infinite delay," Nonlinear Analysis: Theory, Methods \& Applications, vol. 71, no. 12, pp. 6029-6037, 2009.

[67] C. Cuevas and H. Henríquez, "Solutions of second order abstract retarded functional differential equations on the line," submitted.

[68] S. Bochner, "Continuous mappings of almost automorphic and almost periodic functions," Proceedings of the National Academy of Sciences of the United States of America, vol. 52, pp. 907-910, 1964.

[69] G. M. N'Guérékata, Topics in Almost Automorphy, Springer, New York, NY, USA, 2005.

[70] G. M. N'Guérékata, Almost Automorphic and Almost Periodic Functions in Abstract Spaces, Kluwer Academic Publishers/Plenum Press, New York, NY, USA, 2001.

[71] G. M. N'Guérékata, “Quelques remarques sur les fonctions asymptotiquement presque automorphes," Les Annales des Sciences Mathématiques du Québec, vol. 7, no. 2, pp. 185-191, 1983.

[72] D. Bugajewski and G. M. N'Guérékata, "On the topological structure of almost automorphic and asymptotically almost automorphic solutions of differential and integral equations in abstract spaces," Nonlinear Analysis: Theory, Methods E Applications, vol. 59, no. 8, pp. 1333-1345, 2004.

[73] T. Diagana and G. M. N'Guérékata, "Almost automorphic solutions to some classes of partial evolution equations," Applied Mathematics Letters, vol. 20, no. 4, pp. 462-466, 2007.

[74] T. Diagana, G. M. N'Guérékata, and N. V. Minh, "Almost automorphic solutions of evolution equations," Proceedings of the American Mathematical Society, vol. 132, no. 11, pp. 3289-3298, 2004.

[75] H.-S. Ding, T.-J. Xiao, and J. Liang, "Asymptotically almost automorphic solutions for some integrodifferential equations with nonlocal initial conditions," Journal of Mathematical Analysis and Applications, vol. 338, no. 1, pp. 141-151, 2008. 
[76] T. Diagana, E. M. Hernández, and J. P. C. dos Santos, “Existence of asymptotically almost automorphic solutions to some abstract partial neutral integro-differential equations," Nonlinear Analysis: Theory, Methods E Applications, vol. 71, no. 1-2, pp. 248-257, 2009.

[77] R. H. Martin, Jr., Nonlinear Operators and Differential Equations in Banach Spaces, Robert E. Krieger, Melbourne, Fla, USA, 1987.

[78] J. Simon, “Compact sets in the space $L^{p}(0, T ; ß)$, , Annali di Matematica Pura ed Applicata. Serie Quarta, vol. 146, pp. 65-96, 1987. 